\title{
Perceptually-motivated shape exaggeration
}

\author{
Xin Zhang • Wei Chen · Jing Fang • Rui Wang • \\ Qunsheng Peng
}

Published online: 14 April 2010

(C) Springer-Verlag 2010

\begin{abstract}
Image communication would appear more efficient if the visual cases of the concerned parts can be enhanced. One way to achieve this is by local shape exaggeration in rendering. In this paper, we present an interactive scheme for controllable local shape exaggeration. Our approach achieves local, direct, and consistent appearance enhancement by modifying the surface orientation in an intuitive and globally optimized manner with sparse userspecified constraints. Compared with previous approaches, the main contribution of this paper is the introduction of adaptive exaggeration function (AEF), which is capable of modulating the extent of detail enhancement to obtain a satisfactory shape exaggeration result. The AEF model is derived based on a series of experiments. We complement our new approach with a variety of examples, user studies, and provide comparisons with recent approaches.
\end{abstract}

Keywords Shape exaggeration - Weighted least square · Adaptive exaggeration function

\section{Introduction}

Visual communication through computer graphics would greatly benefit from shape exaggeration, which is essentially a feature enhancement process by selectively amplifying or suppressing surface details [1, 2]. In the past decade, much progress has been made toward this goal, like tone shading [3], line drawing [4, 5], and local contrast enhancement [6]. Most existing interactive systems offer facilities

X. Zhang $\cdot$ W. Chen $\cdot$ J. Fang $\cdot$ R. Wang $(\bowtie) \cdot$ Q. Peng

State Key Lab of CAD\&CG, Zhejiang University,

Hangzhou 310027, P.R. China

e-mail: rwang@cad.zju.edu.cn for users to choose effective shape shading algorithms [6$9,11]$, adjust the lighting and viewing parameters [12, 13], change the surface geometry [14-16], warp the environment lighting [17], or scale the radiance [18] so as to provide a better visual interpretation of the object to be rendered. In many applications, such as the digital recovery of archaeological and architectural information [19], medical illustration [20], and computational aesthetics [21, 22], it is desirable to selectively emphasize key regions meanwhile allowing users to control the exaggeration degree that would enhance the visual cues without introducing visual artifacts.

Similar situations exist in computational photography applications that require fine-grained correction and enhancement of images. Recent efforts have enabled local, direct, and intuitive control over different aspects of the results [23-26]. However, this trend has not yet been efficiently extended to shape exaggeration, partly because shape depiction involves multiple aspects such as viewing, lighting, and shading.

In this paper, we address the challenging problem of controllable local shape exaggeration: the manner and the extent of shape exaggeration are controlled by the users in a perceivable manner with simple yet effective interactions. This scheme would provide the users with much more intuitive control than existing parameter-based shape depiction algorithms. Specifically, we intend to construct a local contrast enhancement approach by modifying the surface orientation in the concerned region. Taking the notations of the 3D Unsharp Masking algorithm [6], our goal is to provide a fine-grained solution $U(S)$ for enhancing a surface signal $S$ :

$U(S)=S+\lambda\left(S-S_{\beta}\right)$

where $S$ represents the surface orientation in our context, $S_{\beta}$ denotes the smoothed version of $S$. 
Our approach controls the effects of $U(s)$ by introducing two efficient parameters. The first is $\beta$, called the smoothing parameter, which is used to modulate the smoothness of $S_{\beta}$. The other one is an AEF $\lambda(S)$ that is a linear function adapted to the local geometric complexity of the underlying model and the smoothing parameter $\beta$. We derive the metric $\lambda(S)$ from a series of psychophysical experiments, and validate the effectiveness of the metric for general models. The kernel of our approach is consistent with the scheme of local contrast enhancement that has been studied in [9]. The way we get an optimized smooth parameter $\beta$ is inspired by the weighted least square (WLS) approach introduced in [27]. We provide a stroke-based interface from $2 \mathrm{D}$ to $3 \mathrm{D}$ to facilitate rapid and intuitive selection of the locally exaggerated regions. The combination of the local adjustment and the local contrast enhancement elicits a new application of the locally controllable interface, and complements existing shape exaggeration approaches with more flexible manipulation and interactive tools.

The expressiveness of (1) has been demonstrated by previous methods like the normal enhancement [9] and the radiance enhancement [6]. Our approach, in contrast, is intended to yield more sophisticated effects by offering additionally direct and local control over the results. This add-on option is logically reasonable and operationally simple.

The rest of this paper is organized as follows. In Sect. 2, we briefly review related work. Our approach is described in Sect. 3. In Sect. 4, we introduce a WLS-based normal smoothing approach. Section 5 presents the procedure of getting an appropriate AEF by means of a series of experiments. Further, Sect. 6 extends our scheme by incorporating the local refinement, followed by the detailed results and comparisons in Sect. 7. We discuss and conclude this paper in Sects. 8 and 9.

\section{Related work}

Exaggerated shading reveals distinctive details by dynamically adjusting the extent of visual interpretation. An efficient yet simple approach [9] modifies the surface orientation to emphasize geometric discontinuity. Likewise, the radiance contrast of a scene can be locally enhanced with a 3D Unsharp Masking method [6]. Ihrke et al. [28] gave an evaluation of 3D Unsharp Masking, but it did not obtain a function to depict the relationship between exaggeration extents and underlying scenes. By optimizing the placement of lights based on the geometry, a locally consistent but globally discrepant shading effect is achieved in [13]. Likewise, the environment lighting is warped around main surface features to enhance the shape depiction of 3D objects. Another lighting based optimization approach [12] designs a light for each vertex to achieve maximum local contrast.
While these methods take into account the adaptivity of the underlying scene as well as the lighting and viewing parameters, and achieve appealing results, a direct and intuitive control by the users is desirable. For instance, a new viewindependent filter [14] is used to persuade visual attention through patchwise geometry modification. Our approach advances previous shape exaggeration approaches by allowing the users to design distinctive shape illustration without explicitly changing the geometry.

Line drawing is attractive in that it outlines a $3 \mathrm{D}$ object with the simplest representation to achieve an extremely abstractive shape illustration. Many methods express the shape with a variety of view-dependent curves including the contours [29-31], suggestive contours [4, 32], highlight lines [33], apparent ridges [34], and photic extremum lines [35]. Alternatively, the shape exaggeration through line drawing can be view-independent, such as the ridges and valleys [36-38], and the demarcating curves [8]. We regard line drawing as a special type of shape exaggeration.

The WLS is an optimization framework that was first introduced to deblur noisy images [39]. It interprets the image manipulation as an energy minimization problem concerning the adaptive adjustments to the zero- and first-order continuities. By varying the domain of each of the two items, it is capable of tackling a variety of image editing tasks, including but not limited to colorization [24], tone mapping [27], image matting [25], and edge-preserving tone and image manipulation [40]. Our approach takes advantage of this framework, yet is unique in that it pioneers in terms of locally controllable 3D shape depiction.

\section{Method}

The kernel of our approach is an adaptive exaggeration function (AEF) that is feasible for varied shape exaggeration methods such as the 3D Unsharp Masking [6] and the normal enhancement algorithm [9]. In this paper, we demonstrate the efficiency of the AEF under the framework of the normal enhancement scheme [9]. The significant improvement over the standard approach is the ways of localizing the exaggeration area and controlling the exaggeration strength. This is accomplished by introducing a smoothing parameter $\beta$ to compute a base surface. Users only need to scribble on the target surface to indicate the effected area and the indications are propagated to the desired domain by solving a WLS-based optimization equation. A shape-relevant AEF $\lambda(\cdot)$ adapted to the local shape complexity and the smoothing parameter is designed for better control of the shape exaggeration with (1). Our system also supports local refinement to allow for more accurate local control. The entire process is shown in Fig. 1 with the following highlights: 


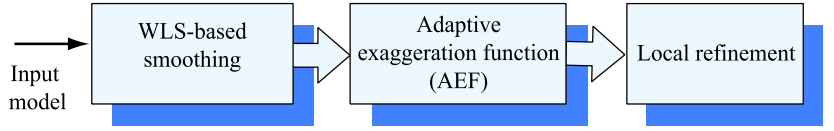

Fig. 1 An overview of our framework

Simple user interface The users can freely specify regions of interest by drawing strokes on the target surface. In the meantime, the users specify the exaggeration parameters to determine the extent of amplifying or suppressing the visual cues of selected regions. The shape exaggeration can be performed either globally or locally.

Global WLS optimization The WLS optimization framework supports globally or locally controllable smoothing of the surface signal. Global smoothing decomposes the model into a low frequency (base) layer and a high frequency (detail) layer by taking the intrinsic surface property into account. For the local smoothing, a set of constraints are specified and propagated into the entire domain to get an optimized layer decomposition.

Adaptive Exaggeration Function (AEF) We conduct a series of experiments that study how object geometry, the smoothing parameter $\beta$, and the extent of detail enhancement interact to influence shape exaggeration. We then derive an $\mathrm{AEF}$, a novel metric to predict how to determine the exaggeration extent to obtain a satisfactory shape exaggeration according to different geometries and smoothing parameters. We also run a confirmatory study to validate the effectiveness of our metric.

Various rendering settings Our approach can be applied to a variety of rendering settings, using object materials ranging from diffuse to specular, and providing styles that range from photorealistic to non-photorealistic.

\section{WLS-based normal smoothing}

The WLS optimization is essentially a smoothing operator, and is applied onto the surface orientation in our approach. Suppose that the input objects are triangular meshes. For each vertex $\mathbf{v}$, we define an interest metric $\delta_{v}$ (see Fig. 2):

$\delta_{v}=\left\|\mathbf{n}_{v}-\frac{\sum_{\mathbf{p} \in N(v)} W_{c}(\|\mathbf{v}-\mathbf{p}\|) W_{f}\left(\left\langle\mathbf{n}_{v}, \mathbf{v}-\mathbf{p}\right\rangle\right) \mathbf{n}_{p}}{\sum_{\mathbf{p} \in N(v)} W_{c}(\|\mathbf{v}-\mathbf{p}\|) W_{f}\left(\left\langle\mathbf{n}_{v}, \mathbf{v}-\mathbf{p}\right\rangle\right)}\right\|^{2}$

Here, $N(v)$ denotes the neighborhood of $\mathbf{v}$, i.e., the dots shown in Fig. 2. $T$ is the tangent plane at $\mathbf{v}, \mathbf{n}_{v}$, and $\mathbf{n}_{p}$ are the normals corresponding to $\mathbf{v}$ and p. $W_{c}(x)$ and $W_{f}(x)$

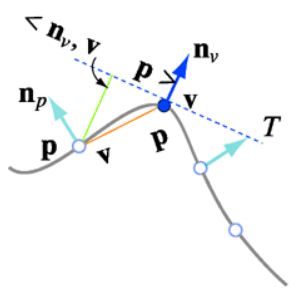

(a)

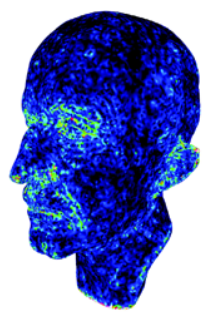

(b)

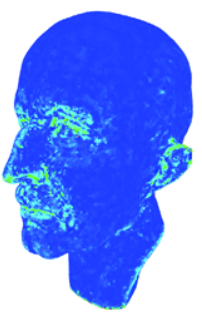

(c)
Color key: black $=0$

Fig. 2 (a) Illustration of the interest metric for a vertex $\mathbf{v}$; (b) The computed interest metric for the Max Plank model; (c) The mean curvature shading of the Max Plank model. The Rainbow color map is used for (b) and (c)

are the weights used in the bilateral filter [10]. Specifically, $W_{c}(x)=e^{-x^{2} / 2 \sigma_{c}^{2}}$ is the closeness smoothing function with a parameter $\sigma_{c}$ that gives larger weights to vertices closer to $\mathbf{v} ; W_{f}(x)=e^{-x^{2} / 2 \sigma_{f}^{2}}$ is a weighting function associated with a parameter $\sigma_{f}$ that penalizes a large variation in terms of the height from the local tangent plane.

Given a model, we group all vertex normals into a long vector $\mathbf{g}=\left(\mathbf{g}_{0}, \mathbf{g}_{1}, \ldots, \mathbf{g}_{i}, \ldots\right)$, and seek to compute another long vector $\mathbf{u}=\left(\mathbf{u}_{0}, \mathbf{u}_{1}, \ldots, \mathbf{u}_{i}, \ldots\right)$ that is as smooth as possible everywhere. Here, $\mathbf{g}$ and $\mathbf{u}$ are two sets of surface normals over the vertex set $\left(\mathbf{v}_{0}, \mathbf{v}_{1}, \ldots, \mathbf{v}_{i}, \ldots\right)$ corresponding to $S$ and $S_{\beta}$ in (1). The computation of $\mathbf{u}$ is formulated as minimizing a WLS energy:

$E=\sum_{i}\left(\mathbf{u}_{i}-\mathbf{g}_{i}\right)^{2}+\beta a_{g_{i}} \delta_{u_{i}}$

where $\mathbf{u}_{i}$ and $\mathbf{g}_{i}$ denote a pair of normals in $\mathbf{u}$ and $\mathbf{g}$. The second term implies the normal adjustment of the target surface in the concerned region, where the smoothing parameter $\beta$ defines the smoothing effort: increasing $\beta$ results in a smoother vector $\mathbf{u}$, and vice versa. The weight $a_{g_{i}}$ is set to be $\left(\delta_{g_{i}}+\varepsilon\right)^{-1}$, and $\varepsilon$ is a small constant (typically 0.0001 ) that prevents division by zero in areas where $\mathbf{g}$ is smooth. Equation (3) can be rewritten into a matrix form:

$E=(\mathbf{u}-\mathbf{g})^{\mathrm{T}}(\mathbf{u}-\mathbf{g})+\beta \mathbf{u}^{\mathrm{T}} \mathbf{D}^{\mathrm{T}} \mathbf{A D} \mathbf{u}$

where $\mathbf{A}$ is a diagonal matrix which corresponds to the set of $a_{g_{i}}$, $\mathbf{D}$ is an $n \times n$ sparse matrix whose element $d_{i j}$ is dependent on the interest metric $\delta_{v}$ :

$d_{i j}= \begin{cases}1, & \text { if } i=j, \\ -W_{i j}, & \text { if } \mathbf{v}_{j} \in N\left(v_{i}\right), \\ 0, & \text { otherwise }\end{cases}$

with

$W_{i j}=\frac{W_{c}\left(\left\|\mathbf{v}_{i}-\mathbf{v}_{j}\right\|\right) W_{f}\left(\left\langle\mathbf{g}_{i}, \mathbf{v}_{i}-\mathbf{v}_{j}\right\rangle\right)}{\sum_{\mathbf{v}_{j} \in N\left(v_{i}\right)} W_{c}\left(\left\|\mathbf{v}_{i}-\mathbf{v}_{j}\right\|\right) W_{f}\left(\left\langle\mathbf{g}_{i}, \mathbf{v}_{i}-\mathbf{v}_{j}\right\rangle\right)}$ 


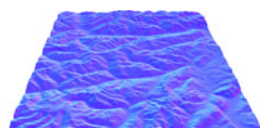

(a)

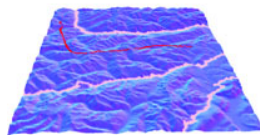

(d)

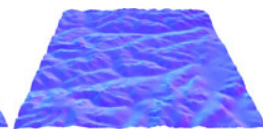

(b)

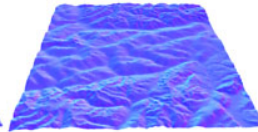

(e)

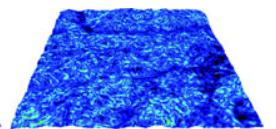

(c)

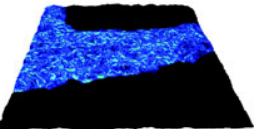

(f)
Fig. 3 WLS-based smoothing with $\beta=0.5$ applied to the Dolomiti model; (a) The input model; (d) A stroke drawn by the users. The model is divided into four patches, which are bounded with pink curves. (b), (e) The extracted base layers; (c), (f) The detail layers. In all cases, each surface normal is illustrated with a RGB-triple color

The vector $\mathbf{u}$ that minimizes (3) is uniquely defined as the solution of the following linear system:

$\mathbf{L u}=\mathbf{g}$

where $\mathbf{L}=\mathbf{I}+\beta \mathbf{D}^{\mathrm{T}} \mathbf{A D}$, and $\mathbf{I}$ is an $n \times n$ identity matrix.

By solving the linear system, we get the base layer $\mathbf{u}$ of the input signal $\mathbf{g}$ and the detail layer $\mathbf{d}=\mathbf{g}-\mathbf{u}$, as depicted in Fig. 3(a-c). In terms of the normal enhancement, (1) becomes:

$U\left(\mathbf{g}_{i}\right)=\mathbf{u}_{i}+\lambda \mathbf{d}_{i}$

To facilitate efficient local specification, we first divide the model into a sequence of patches [49]. The segmentation allows the users to freely indicate regions of interest with a stroke-based interface. We set the regions that are not selected as a set of soft constraints in the WLS optimization framework, and set $\beta=0$ in these regions. Thus, we can obtain a matrix $\mathbf{M}$ as follows:

$\mathbf{M}_{i j}^{*}= \begin{cases}\mathbf{L}_{i j}^{*}, & \text { if } \mathbf{v}_{i} \in V^{*}, \\ 1, & \text { if } \mathbf{v}_{i} \notin V^{*} \text { and } i=j, \\ 0, & \text { otherwise. }\end{cases}$

where the superscript ${ }^{*}$ denotes the parts corresponding to the selected surface patches, and $V^{*}$ is the vertex set in the selected regions.

The effect of the local WLS-based smoothing is distinctive, as demonstrated in Fig. 3(d-f). It faithfully reflects the user intention meanwhile making the results consistent along the region boundary.

\section{Adaptive exaggeration function}

A straightforward implementation of the local contrast enhancement through (1) is to modulate $\lambda$ to get exaggerated shading. However, it is not always the ideal case when a constant parameter is employed. Often, this kind of adaptivity is desirable in many perception-related applications such

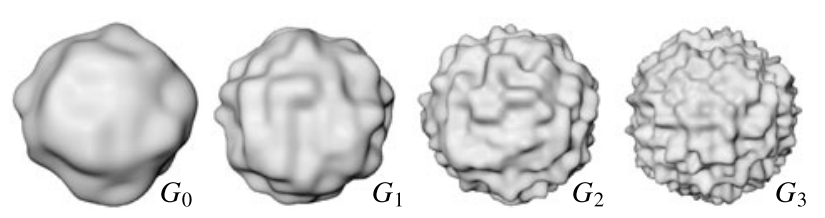

Fig. 4 The test objects used in our experiment

as image manipulation, tone mapping, and image matting. While the user can easily determine where the details should be amplified, suppressed, or unchanged, adjusting the exaggeration parameter patch by patch is tedious. It would be more desirable to have an adaptive scheme to automatically decide the extent of the shape exaggeration.

\subsection{Stimuli}

A set of images were created to allow us to explore the relationship between the geometry $g$, the smoothing parameter $\beta$ and the exaggeration extent $\lambda$. Figure 4 shows the test objects. They are combined with some bumpy ball-like objects. The following paragraphs describe the objects and rendering parameters we used to generate the images.

Geometry: We used the method introduced in [41] to create four object geometries $\left(G_{0}, G_{1}, G_{2}, G_{3}\right)$, as shown in Fig. 4. We chose these geometries because: 1) they facilitate effective analysis of the influences of geometry on the appearance; and 2) they have been widely used in the shape perception literature for similar geometries [41-44].

The smoothing parameter: The smoothing parameter $\beta$ defines the smoothing degree: increasing $\beta$ results in a smoother vector $\mathbf{u}$, and vice versa. We chose four values: $0.1,0.3,0.5,0.7$, to span a significant range of low-to-highsmooth base layer, and to understand the role of the smoothing parameter $\beta$.

Illumination and material: Recent studies have demonstrated the importance of illumination and material to the perception of shape $[45,46]$. To keep our study conservative and tractable, we used the Phong shading model and a single light source. The parameters for illumination were set to be the same as those of [47], and were proved to be the best for shape cognition in [47]. We chose the Phong shading model because it isolates shading from other cues to shape exaggeration, and is adequate for studying the exact relationship between the geometry $g$, the smoothing parameter $\beta$, and the exaggeration extent $\lambda$.

\subsection{Experiment}

Our goal is to explore the relationship between the extent $\lambda$ and shape exaggeration effects with respect to different 


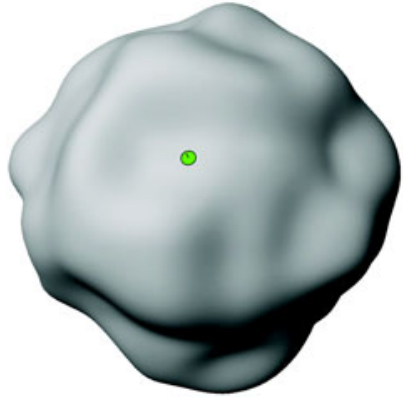

(a)

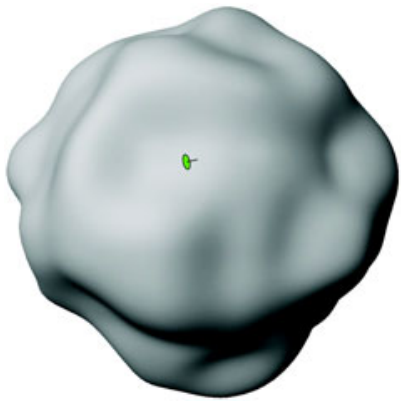

(b)
Fig. 5 Instructional example. (a) Good setting; (b) bad setting

geometries and smoothing parameters. Our experiment is based on the gauge placement protocol described by Koenderink et al. [29]. Data is collected from a total of 14 subjects participating for pay. They have normal or correctedto-normal vision, and are not familiar with the goals of this study. Subjects are shown a series of gauges in random order and are asked to place each gauge correctly before moving on to the next. The subjects have no control over the position of the gauge, but its orientation. Each gauge is drawn as a small ellipse representing a disc together with a single line indicating the normal of the disc. The gauges are superimposed over the images and colored green for visibility (Fig. 5). To avoid cuing the subjects to shape, the gauges do not penetrate or interact with the 3D model. The initial orientations of the gauges are randomly set.

At the beginning of the experiment, subjects are shown a simple example shape that is not used for data collection. The shape has examples of good and bad placements (Fig. 5). Each time the subjects start a session, they are allowed to practice orienting gauges on the example shape before moving on to the actual task. Subjects are asked to orient the gauge by dragging with the mouse, and to advance to the next gauge by pressing the space bar.

For each object and the smoothing parameter, we created a series of images with ten different $\lambda$ ranging from 0.0 to 4.0. During the experiment, 160 images were rendered by using the Phong shading model (4 geometries $\times 4$ smoothing parameters $\times 10$ exaggeration extents). The study was conducted on a 22-inch LCD display with a resolution of $1680 \times 1050$, located at a distance of 33 inches, approximately 30 degrees viewing angle horizontally. The complete session took approximately one hour.

We use the root mean square (RMS) method to measure whether the subjects understand the shape correctly. The smaller the RMS value is, the more correctly the subjects understand it. For each object and smoothing parameter, we choose the $\lambda$ which results in the smallest RMS value. It is regarded as the best exaggeration degree under this condition.

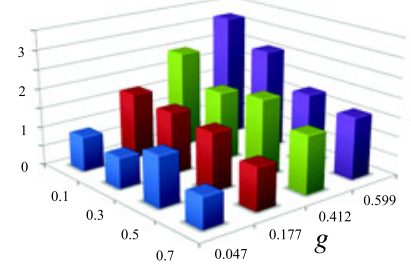

(a)

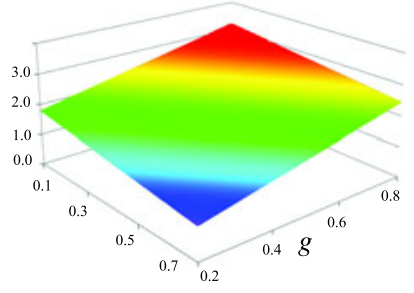

(b)
Fig. 6 Result of the user study. (a) The result of data analysis; (b) the resulting plane that fits the optimal parameters in (a)

The best $\lambda$ for each object and smoothing parameter are plotted in Fig. 6(a). It is apparent that the best exaggeration effects with different $g$ and $\beta$ are achieved with various $\lambda$. In addition, $\lambda$ increases in proportion to the increasing in $g$, and in proportion to the fall in $\beta$. We performed the ANOVA test to demonstrate the availability of our experiment $(F(2,16)=1.916, p<0.01)$.

\subsection{Defining the metric}

With the findings of our user study, we derive a metric to compute an appropriate adaptive exaggeration extent under given geometry complexity and smoothing parameter.

Firstly, we need a way to characterize a surface. Many characterizations of geometry are possible $[4,48]$. We introduce a novel local complexity descriptor. When speaking of a surface $\Phi$ in the following, we mean the discrete set of points from the mesh. Let $q_{i}$ be the $k$-nearest neighbors of a point $p \in \Phi$, and let $n_{p}$ and $n_{q_{i}}$ be the surface normal vectors at these points. We define the local variation $e_{k}(p)$ at $p$ as

$e_{k}(p)=\frac{1}{k} \sum_{i=1}^{k}\left\|n_{p}-n_{q_{i}}\right\|^{2}$

We integrate the local variation over the local neighborhood $N_{r}(p)=S_{r}(p) \cap \Phi$,

$C_{k, r}(p)=\frac{1}{\left|N_{r}(p)\right|} \sum_{q \in N_{r}(p)} e_{k}(q)$

where $S_{r}(p)$ is a sphere of radius $r$ centered at $p . C_{k, r}(p)$ is called the local complexity at $p$. The characteristic value $C_{k, r}(p)$ varies with respect to $k$ and $r$. To ensure that $C_{k, r}(p)$ reflects the actual surface property, a suitable $k$ and $r$ should be chosen. In this paper, we set $k$ to be 50 and $r$ to be four folds to the average length of the model. The average surface complexity for $G_{0}$ to $G_{3}$ were $\{0.047,0.177,0.412,0.599\}$.

We used the least square method to fit a linear function to the parameters of the best effects, as shown in Fig. 6(a). Figure 6 (b) shows the computed plane. Given a point $(c, r, e)$ 


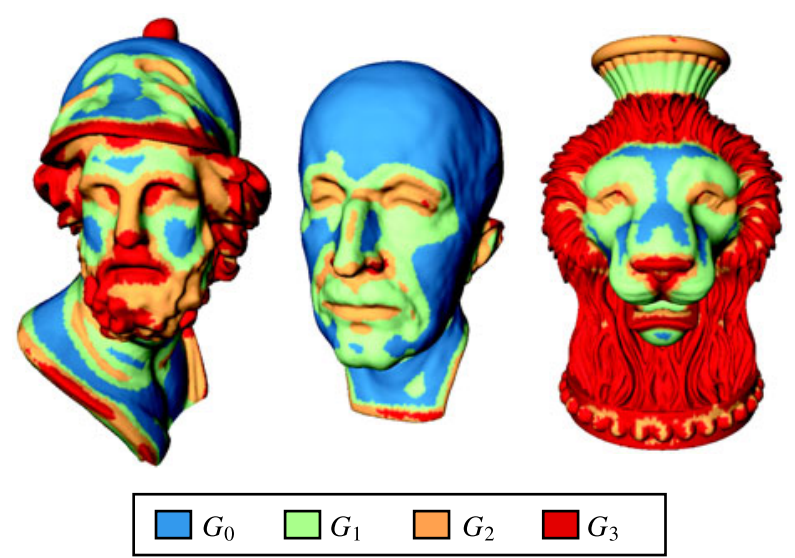

Fig. 7 Local complexity measure for the Ajax, Max Plank, and Lionvase models. The local complexity at each vertex is depicted with a selected color representing a test object in $\left(G_{0}, G_{1}, G_{2}, G_{3}\right)$ whose average complexity is closest to the local complexity

in the space of $(g, \beta, \lambda)$, the equation of the plane $P$ is

$$
2.356 c-1.2625 r-e+1.4463=0
$$

\subsection{Generalizing the metric to other models}

To apply our metric to models with arbitrary geometries and smoothing parameters, we need to project these properties into their corresponding positions in $P$. In particular, the smoothing parameter is chosen by users. We only need to focus on the projection of the geometry complexity.

We compute the local complexity at each vertex with (11), and compute $\lambda$ with (12). Figure 7 illustrates the local complexity at each vertex in the Ajax, Max Plank, and Lionvase models.

\subsection{Metric validation}

To test how well our metric works on other objects, we created new images using 10 additional models. We used the Standford Bunny, Gargo, Lionhead, Fish, Lionvase, Ajax, Guadalupe, Golf Ball, Elephant, and Max Plank models for examination.

We performed another experiment with 14 additional subjects participating for pay. Overall, we created 150 trials (images), of which 15 trials for each model. Among each set of 15 trials, one trial was exaggerated adaptively with our metric, and other 14 trials were exaggerated uniformly with different extents ranging from 0.0 to 4.0. The physical setup was the same as the one explained in Sect. 5.2. The entire procedure took approximately one hour. The images generated by the Ajax model were put at the start of the user study to allow the subjects to acquire experience, and the data of the model was not included in the final analysis.
Table 1 Results of the user study in metric validation

\begin{tabular}{ll}
\hline Test objects & Order/total number \\
\hline Stanford Bunny & $5 / 15$ \\
Lionhead & $1 / 15$ \\
Golf Ball & $4 / 15$ \\
Elephant & $3 / 15$ \\
Fish & $5 / 15$ \\
Gargo & $6 / 15$ \\
MaxPlank & $3 / 15$ \\
Lionvase & $5 / 15$ \\
Rabbit & $5 / 15$ \\
\hline
\end{tabular}

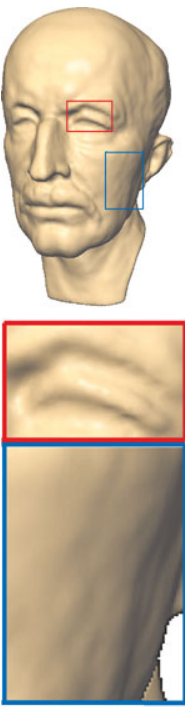

(a)
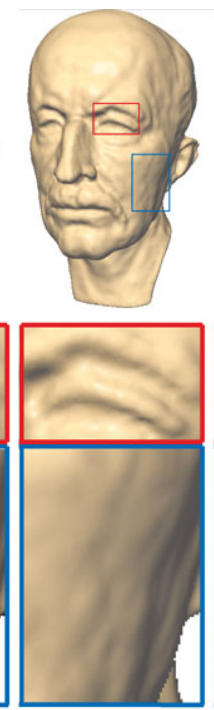

(b)
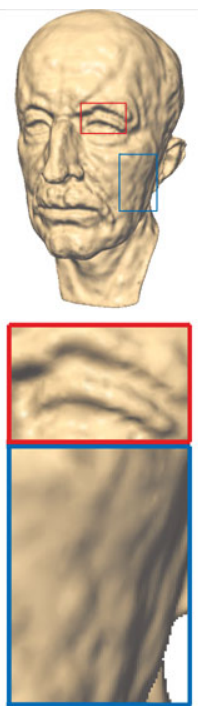

(c)
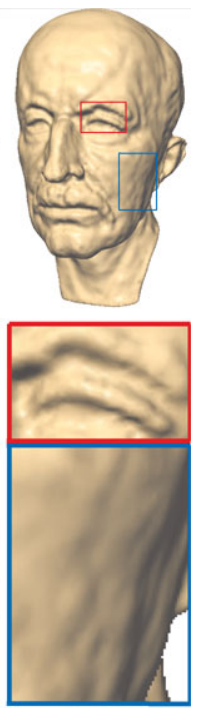

(d)
Fig. 8 Applying our metric to the Max Plank model. (a) The input model; (b) the uniformly exaggerated effect with $\lambda=0.6$; (c) the uniformly exaggerated effect with $\lambda=2.0 ;(\mathbf{d})$ the adaptively exaggerated effect. In all cases, the smoothing parameter $\beta=0.3$

We use RMS method to analyze the experiment data. By ordering the RMS value, we can see whether the adaptive exaggerated effects are understood by subjects correctly. The results for the user study are summarized in Table 1. In the second column, there are two numbers. The first number is the order number of the adaptive exaggeration effect among all effects of the object, while the second one is the total number. The order numbers of mostly models are small. We conclude that our metric is effective in producing satisfactory shape exaggeration automatically. Figure 8 shows the effect of the adaptive shape exaggeration scheme, and the comparison with uniform exaggeration scheme. In Fig. 8(c), both the eye and face regions demonstrate stronger exaggerations than those in (b). In contrast, our adaptive scheme yields strong exaggeration in the eye region and weak exag- 


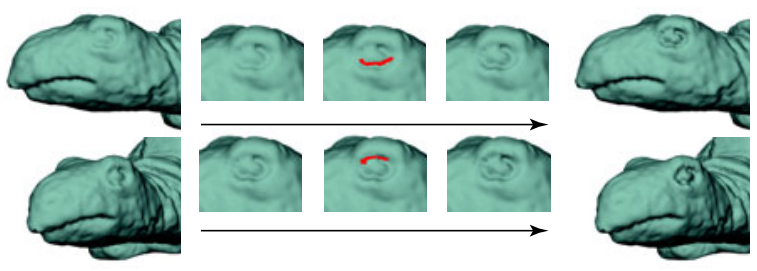

Fig. 9 Local enhancement on the eyes of the Turtle model. Left: the input model; Middle: the exaggeration is performed on the region around the red strokes; Right: the exaggerated results

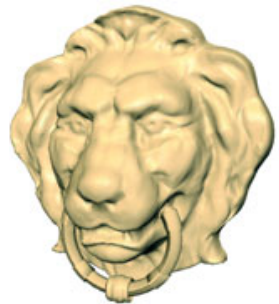

(a)

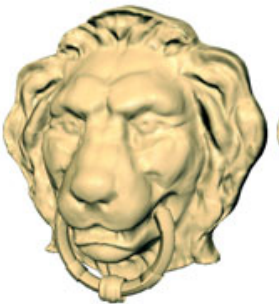

(b)

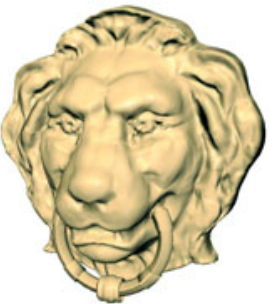

(c)
Fig. 10 Results of the Lionhead model. (a) The input model; (b) Local exaggeration on the hair; (c) Additional local editing to (b) for enhancing two eyes. In all cases, the effects are implemented with $\beta=0.3$

geration in the face region, which are achieved by the uniform exaggeration scheme under different $\lambda$ separately.

\section{Local refinement}

In addition to the WLS-based globally and locally controllable normal smoothing schemes applied to the surface in the entire domain, our system supports local refinement in a small region that means a small-sized neighborhood of userspecified strokes is regarded as the computation domain. In our experiments, a two-ring or three-ring neighborhood of the vertices covered by the strokes yields appropriate results. To perform the WLS-based smoothing locally, we use the $K$-Nearest-Neighbor (KNN) method to select a number of the nearest points (in our experiments, 200) in the neighborhood of the strokes, and assign them with a user-adjustable smoothing parameter. Other points in the neighborhood are set as the soft constraints. Thus, the computational expense is greatly reduced, facilitating real-time local refinement. Figure 9 and Fig. 10(c) depict the exaggeration effects on the eyes of the Turtle and Lionhead models, respectively.

\section{Results}

We implemented the proposed approach on a PC equipped with Intel Core 2 Quad Q9550 $2.83 \mathrm{GHz}$ CPU, 4G host memory, and Nvidia WinFast GTX 280 graphics card. Table 2 reports the experimental statistics, including the number of vertex $(\# \mathrm{~V})$, Triangle (\#T) and the time for handling
Table 2 Performance statistics for a sequence of models

\begin{tabular}{lrrrll}
\hline Data & $\# \mathrm{~V}$ & $\# \mathrm{~T}$ & $\mathrm{TS}(\mathrm{s})$ & Building & Solving \\
\hline Lionhead & $12 \mathrm{~K}$ & $22 \mathrm{~K}$ & 0.84 & $26 \%$ & $74 \%$ \\
Lionvase & $20 \mathrm{~K}$ & $40 \mathrm{~K}$ & 1.70 & $28 \%$ & $72 \%$ \\
Ajax & $23 \mathrm{~K}$ & $45 \mathrm{~K}$ & 2.03 & $29 \%$ & $71 \%$ \\
Fish & $25 \mathrm{~K}$ & $50 \mathrm{~K}$ & 2.40 & $31 \%$ & $69 \%$ \\
Dolomiti & $25 \mathrm{k}$ & $50 \mathrm{~K}$ & 2.33 & $32 \%$ & $68 \%$ \\
Crater & $25 \mathrm{~K}$ & $50 \mathrm{~K}$ & 2.39 & $31 \%$ & $69 \%$ \\
Golfball & $31 \mathrm{~K}$ & $61 \mathrm{~K}$ & 3.55 & $26 \%$ & $74 \%$ \\
MaxPlank & $49 \mathrm{~K}$ & $98 \mathrm{~K}$ & 7.23 & $24 \%$ & $76 \%$ \\
Turtle & $134 \mathrm{~K}$ & $268 \mathrm{~K}$ & 15.6 & $20 \%$ & $80 \%$ \\
Armadillo & $150 \mathrm{~K}$ & $300 \mathrm{~K}$ & 16.9 & $25 \%$ & $75 \%$ \\
\hline
\end{tabular}

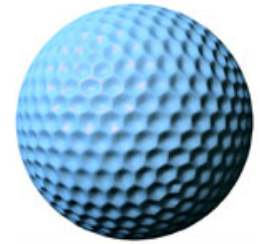

(a)

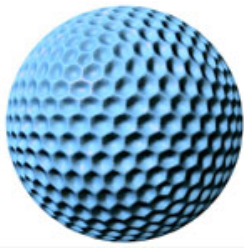

(b)

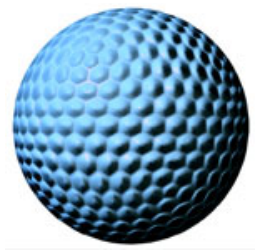

(c)
Fig. 11 Global exaggeration on the golf ball model. (a) The input model; (b) global exaggeration with $\beta=0.3$; (c) global exaggeration with $\lambda(\cdot)=-5.0$

(TS) the models presented in this paper. The total WLS optimization time is dominated by the parts for building and solving the linear system. We used the Intel Math Kernel Library to solve the linear system in our implementation. The "building" and "solving" columns report the time percentages with respect to TS.

Figure 10(b) shows the local exaggeration results on the hair part of the Lionhead model. Additionally, we refine the eyes locally based on (b), as shown in Fig. 10(c).

In Fig. 11, we show a global exaggeration result on the Golfball model, for which the AEF $\lambda(\cdot)$ is set to be a negative constant, as shown in Fig. 11(c). This causes the convex regions to be concave, and vice versa.

Figure 12 displays two exaggerated results that enhance different parts of the Fish model. Figure 12(b) emphasizes the head, fishtail, and body parts, while Fig. 12(d) emphasizes the fin parts. In each case, shading across the region boundaries are kept continuous. The effects of the global and local approaches are compared in Fig. 13. By presenting different regions with various visual cues, the terrain visualization can be task-tailored and user-adaptable. Another interesting example is demonstrated in Fig. 14, where the silhouettes become more apparent for the mustache and hat parts after shape exaggeration.

Figure 15 illustrates the exaggeration results on the Armadillo model with our method in photorealistic rendering style. We use wavelet based precomputed radiance transfer 


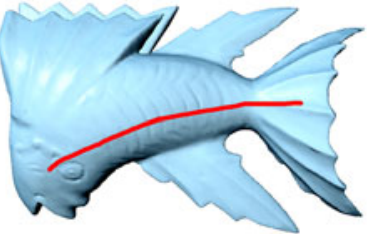

(a)

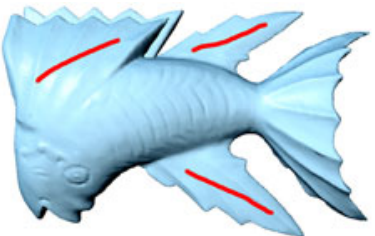

(c)

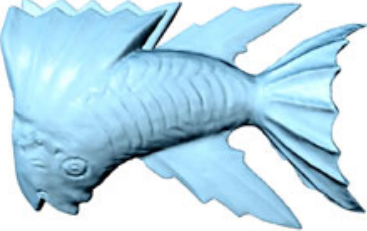

(b)

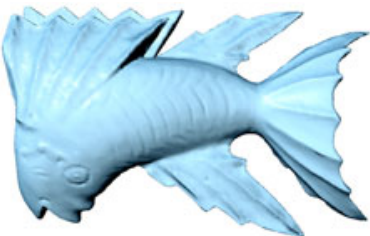

(d)
Fig. 12 Results of the Fish Model. (a) and (c) shows the user strokes; (b) the head, body, and fishtail parts are enhanced; (d) the fin parts are enhanced. In all cases, the effects are implemented with $\beta=0.5$

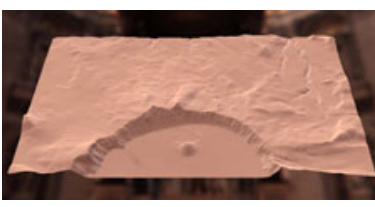

(a)

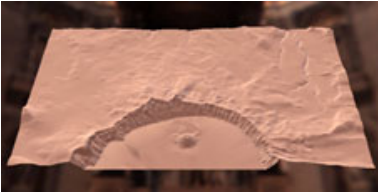

(c)

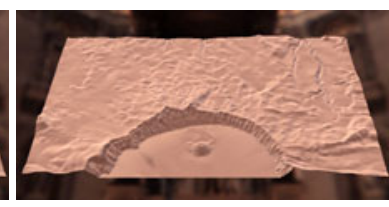

(b)

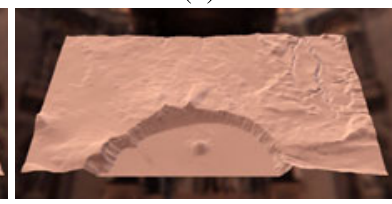

(d)
Fig. 13 Shape exaggeration of the Crater Terrain model in photorealistic rendering style. (a) The input model; (b) Global exaggeration; (c) Local exaggeration on the bottom part; (d) Local exaggeration on the upper right part. In all cases, the effects are implemented with $\beta=0.5$

method [50] to render 3D objects. Our exaggeration results also can be extended to non-photorealistic rendering style, as shown in Fig. 16. The line drawings clearly demonstrate that the feature enhancement is achieved with the globally controlled exaggeration technique.

\section{Discussion}

Our approach enhances surface features with various materials, illuminations, and styles. It may produce results similar to exaggerated shading [12], 3D Unsharp Masking [6] or light warping approach [17]. Compared to exaggerated shading [12], our approach exaggerates surface details with a much wider range of materials and lightings. Furthermore, exaggerated shading requires a time-consuming preprocess, suffers from light direction sensitivity, and tends to flatten the overall shape perception, as shown in Fig. 17. The

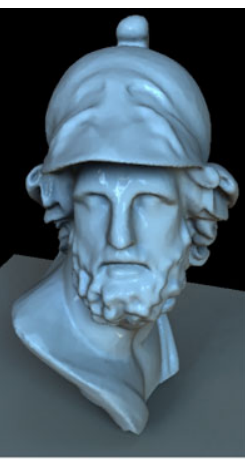

(a)

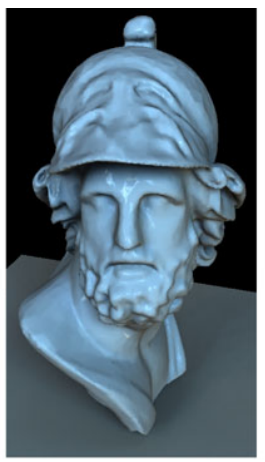

(b)

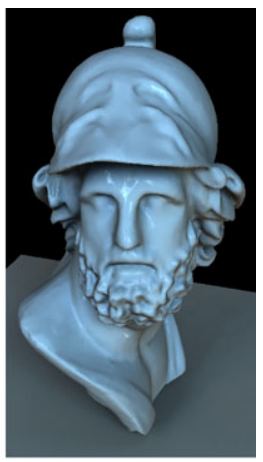

(c)
Fig. 14 Results for the Ajax model in Photorealistic rendering style. (a) The input model; (b) Local exaggeration on the hat part; (c) Local exaggeration on the mustache part. In all cases, the effects are implemented with $\beta=0.3$

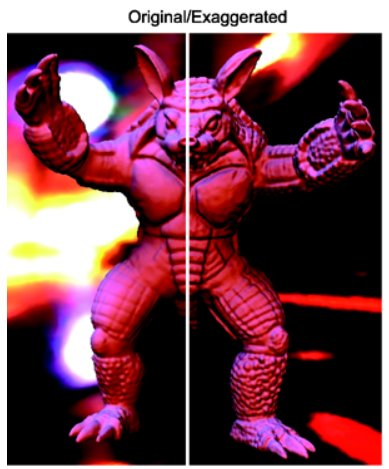

Diffuse material

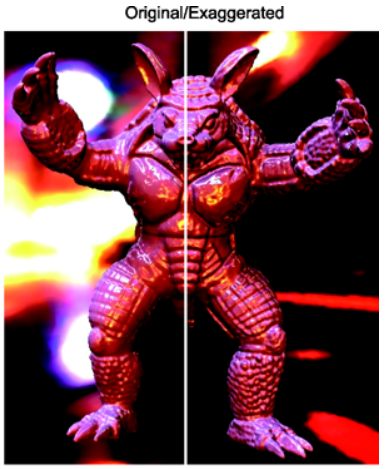

Specular material
Fig. 15 Photorealistic rendering results: the Armadillo model rendered with diffuse and specular materials. Each side of the figure shows original and exaggerated results

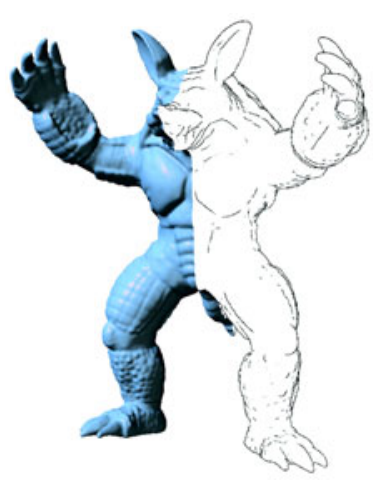

(a)

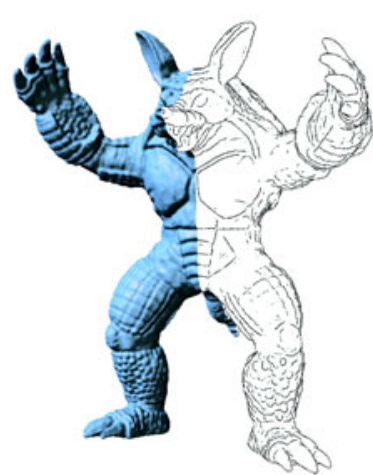

(b)
Fig. 16 Results for the Armadillo model; (a) The input model; (b) Global exaggeration. In both cases, the left part is rendered with Phong shading, while the right part is displayed with line drawing (silhouette and suggestive contours)

right effect shown in Fig. 17 is implemented with our system, while the left was generated by the software described in [12]. The employed parameters are: 0.8 for the contrast 


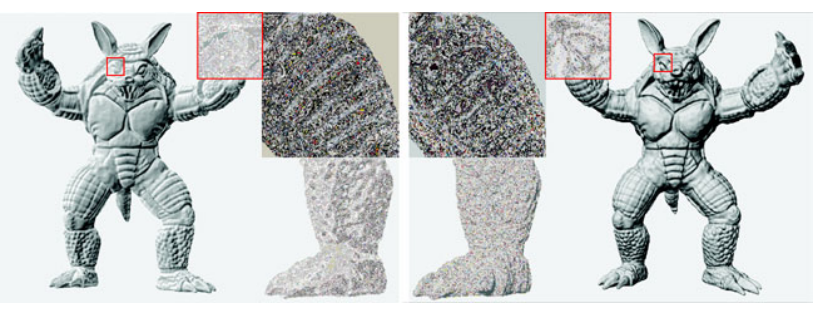

Fig. 17 Result comparison for the Armadillo model. (Left) Exaggerated shading; (right) Our global exaggeration approach with $\beta=0.3$
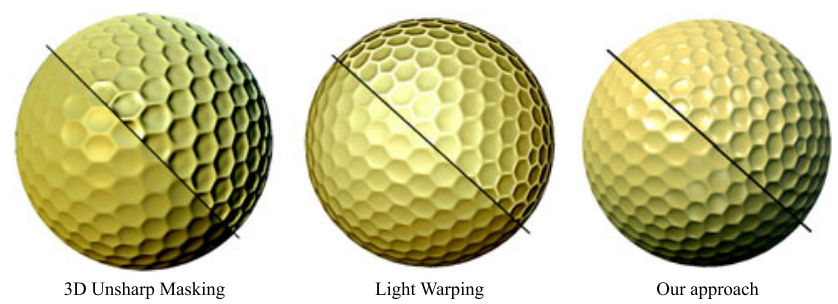

Fig. 18 Result comparison for the golf ball model. (Left) 3D Unsharp Masking method; (middle) Light warping method; (right) Our global exaggeration approach with $\beta=0.3$. Our approach can keep material properties unchanged during exaggeration. Images of previous techniques are extracted from original papers and supplemental materials

of the base layer, 0.2 for the contrast of the exaggerated shading. Overall our method achieves comparable effects as [12], but leads to more appealing results in some regions such as the eyes, the right leg, and the left foot. Compared to 3D Unsharp Masking [6], our system enhances surface features by changing normals, not radiances. Thus, our approach can keep material properties unchanged during exaggeration, while 3D Unsharp Masking increases the radiance contrast so that it may alter material properties, as shown in Fig. 18. Compared to the light warping approach [17], our system can be applied to various rendering styles, while the light warping approach depends on the existence of light variations in the scene. Furthermore, the shape of shadows may be distorted due to the warped light. Besides, our approach is also simpler to control compared to previous work, as it offers only one parameter: the smoothing parameter $\beta$.

Our approach shows some limitations though. First, the performance can be improved, especially on solving the WLS system. We plan to have a GPU implementation of the solver, like the one described by Buatois et al. [51]. Buatois et al. [51] describe a GPU implementation of a more general preconditioned conjugate gradient sparse matrix solver, and report speedups by a factor of 3.2 over a highly optimized CPU implementation. Second, the local exaggerated scheme assumes that the object segmentation has already been done. Concerning this issue, we expect to design a perceptiondriven mesh segmentation algorithm for easier control.

The persuading filter [14] modifies the surface geometry on a local region. While fast and simple, it may lead

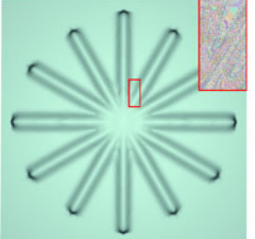

(a)

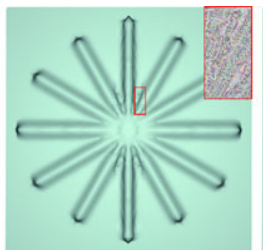

(b)

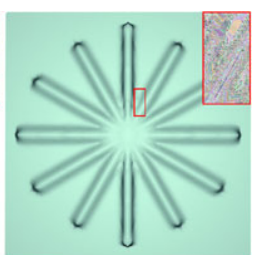

(c)
Fig. 19 Result comparison of the Cross model. (a) Phong shading; (b) Persuade filter; (c) Our local exaggeration approach

to nonsmooth transition among region boundary in some extreme situations. In contrast, our local exaggeration approach achieves visually attractive effect with comparable performance, as shown in Fig. 19.

\section{Conclusion}

We have presented an interactive tool for local, intuitive, and fine-grained control over exaggerated shading. Challenges in this include how to determine the scale of local contrast and how to achieve perceptually effective results. Whether our results demonstrate a promising shape illustration may be a matter of taste. But the experimental results do indicate that our results tend to approximate the user intentions, and that it is probably intractable to get the similar results with existing shape exaggeration methods.

There are some interesting topics valuable for future exploration. The current solution for measuring the local complexity is independent of illumination and material. Additional consideration of the illumination and material configuration may lead to more plausible results. Moreover, the proposed AEF is linear, and we expect to derive a better metric by using high order functions. We also plan to design a perception-driven mesh segmentation algorithm for easier control.

Acknowledgements This research is supported by National Grand Foundation Research 973 Program of China under Grant No. 2009CB320802, National Natural Science Foundation of China Under Grant No. 60970020 and No. 60873123. We would like to thank Mr. Pengfei Xu and Mr. Naiyang Lin for their help.

\section{References}

1. Gooch, B., Gooch, A.: Non-Photorealistic Rendering. A.K. Peters Ltd (2001)

2. Guskov, I., Sweldens, W., Schroder, P.: Multiresolution signal processing for meshes. In: Proceedings of SIGGRAPH 1999, pp. 325-334 (1999)

3. Gooch, A., Gooch, B., Shirley, P., Cohen, E.: A non-photorealistic lighting model for automatic technical illustration. In: Proceedings of SIGGRAPH '98, 31, pp. 447-452 (1998)

4. DeCarlo, D., Finkelstein, A., Rusinkiewicz, S., Santella, A.: Suggestive contours for conveying shape. ACM Trans. Graph. 22(3), $848-855$ (2003) 
5. Cole, F., DeCarlo, D., Finkelstein, A., Kin, K., Morley, K., Santella, A.: Directing gaze in 3D models with stylized focus. In: Eurographics Symposium on Rendering, pp. 377-387 (2006)

6. Ritschel, T., Smith, K., Ihrke, M., Grosch, T., Myszkowski, K., Seidel, H.-P.: 3D unsharp masking for scene coherent enhancement. ACM Trans. Graph. 27(3) (2008)

7. Lee, Y., Markosian, L., Lee, S., Hughes, J.F.: Line drawings via abstracted shading. ACM Trans. Graph. 26(3), 18:1-18:5 (2007)

8. Kolomenkin, M., Shimshoni, I., Tal, A.: Demarcating curves for shape illustration. ACM Trans. Graph. 27(4), 157:1-157:9 (2008)

9. Cignoni, P., Scopigno, R., Tarini, M.: A simple normal enhancement technique for interactive non-photorealistic renderings. Comput. Graph. 25(1), 125-133 (2005)

10. Fleishman, S., Drori, I., Cohen-Or, D.: Bilateral mesh denoising. ACM Trans. Graph. 22(3), 950-953 (2003)

11. Todo, H., Anjyo, K., Baxter, W., Igarashi, T.: Locally controllable stylized shading. ACM Trans. Graph. 26(3), 17:1-17:7 (2007)

12. Rusinkiewicz, S., Burns, M., DeCarlo, D.: Exaggerated shading for depicting shape and detail. ACM Trans. Graph. 25(3), 11991205 (2006)

13. Lee, C.H., Hao, X., Varshney, A.: Geometry-dependent lighting. IEEE Trans. Vis. Comput. Graph. 12(2), 197-207 (2006)

14. Kim, Y., Varshney, A.: Persuading visual attention through geometry. IEEE Trans. Vis. Comput. Graph. 14(4), 772-781 (2008)

15. Kim, B.M., Rossignac, J.: GeoFilter: geometric selection of mesh filter parameters. Comput. Graph. Forum 24(3), 295-302 (2005)

16. Eigensatz, M., Sumner, R.W., Pauly, M.: Curvature-domain shape processing. Comput. Graph. Forum 27(2), 241-250 (2008)

17. Vergne, R., Pacanowski, R., Barla, P., Granier, X., Schlick, C.: Light warping for enhanced surface depiction. ACM Trans. Graph. 28(3) (2009)

18. Vergne, R., Pacanowski, R., Barla, P., Granier, X., Schlick, C.: Radiance scaling for versatile surface enhancement. In: I3D '10: Proc. Symposium on Interactive 3D graphics and games, ACM (2010)

19. van der Maaten, L.J.P., Boon, P.J., Paijmans, J.J., Lange, A.G., Postma, E.O.: Computer vision and machine learning for archaeology. In: Proceedings of Computer Applications and Quantitative Methods in Archaeology, pp. 112-130 (2006)

20. Ebert, D., Sousa, M.C.: Illustrative visualization for medicine and science. In: Proceedings of ACM SIGGRAPH Courses (2006)

21. Strothotte, T., Schlechtweg, S.: Non-Photorealistic Computer Graphics: Modeling, Rendering, and Animation. Morgan Kaufmann, San Mateo (2002)

22. DeCarlo, D., Santella, A.: Stylization and abstraction of photographs. ACM Trans. Graph. 21(3), 769-776 (2002)

23. Pérez, P., Gangnet, M., Blake, A.: Poisson image editing. ACM Trans. Graph. 22(3), 313-318 (2003)

24. Levin, A., Lischinski, D., Weiss, Y.: Colorization using optimization. ACM Trans. Graph. 23(3), 689-694 (2004)

25. Levin, A., Lischinski, D., Weiss, Y.: A closed-form solution to natural image matting. IEEE Trans. Pattern Anal. Mach. Intell. 30(2), 228-242 (2008)

26. Toler-Franklin, C., Finkelstein, A., Rusinkiewicz, S.: Illustration of complex real-world objects using images with normals. Comput. Graph. Forum 26(3), 385-394 (2007)

27. Lischinski, D., Farbman, Z., Uyttendaele, M., Szeliski, R.: Interactive local adjustment of tonal values. ACM Trans. Graph. 25(3), 646-653 (2006)

28. Ihrke, M., Ritschel, T., Smith, K., Grosch, T., Myszkowski, K., Seidel, H.-P.: A perceptual evaluation of (3D) unsharp masking, human vision and electronic imaging XIII. SPIE 72400R72400R-12 (2009)

29. Koenderink, J.J.: What does the occluding contour tell us about solid shape. Perception 13(3) (1984)
30. Gooch, B., Sloan, P.-P.J., Gooch, A., Shirley, P., Riesenfeld, R.: Interactive technical illustration. In: Proceedings of ACM Symposium on Interactive 3D graphics, pp. 31-38 (1999)

31. Hertzmann, A., Zorin, D.: Illustrating smooth surfaces. In: Proceedings of ACM SIGGRAPH 2000, pp. 517-526 (2000)

32. DeCarlo, D., Finkelstein, A., Rusinkiewicz, S.: Interactive rendering of suggestive contours with temporal coherence. In: Proceedings of NPAR, pp. 15-24 (2004)

33. DeCarlo, D., Rusinkiewicz, S.: Highlight lines for conveying shape. In: Proceedings of NPAR, pp. 63-70 (2007)

34. Judd, T., Durand, F., Adelson, E.H., Santella, A.: Apparent ridges for line drawing. ACM Trans. Graph. 26(3), 19:1-19:7 (2007)

35. Xie, X., He, Y., Tian, F., Seah, H.-S., Gu, X., Qin, H.: An effective illustrative visualization framework based on photic extremum lines (PELs). IEEE Trans. Vis. Comput. Graph. 13(6), 1328-1335 (2007)

36. Interrante, V., Fuchs, H., Pizer, S.M.: Enhancing transparent skin surfaces with ridge and valley lines. In: IEEE Visualization, pp. 52-59 (1995)

37. Kalnins, R.D., Markosian, L., Meier, B.J., Kowalski, M.A., Lee, J.C., Davidson, P.L., Webb, M., Hughes, J.F., Finkelstein, A., WYSIWYG, N.P.R.: drawing strokes directly on 3D models. ACM Trans. Graph. 21(3), 755-762 (2002)

38. Ohtake, Y., Belyaev, A., Seidel, H.-P.: Ridge-valley lines on meshes via implicit surface fitting. ACM Trans. Graph. 23(3), 609-612 (2004)

39. Lagendijk, R.L., Biemond, J., Boekee, D.E.: WYSIWYG NPR: Drawing strokes directly on 3D models. IEEE Trans. Acoust. Speech Signal Process. 36(12), 1874-1888 (1988)

40. Farbman, Z., Fattal, R., Lischinski, D., Szeliski, R.: Edgepreserving decompositions for multi-scale tone and detail manipulation. ACM Trans. Graph. 27(3), 1-10 (2008)

41. Ramanarayanan, G., Ferwerda, J.A., Walter, B., Bala, K.: Visual equivalence: towards a new standard for image fidelity. ACM Trans. Graph. 26(3), 76:1-76:11 (2007)

42. Todd, J.T., Mingolla, E.: Perception of surface curvature and direction of illumination from patterns of shading. J. Exp. Psychol. Hum. Percept. Perform. 9(4), 583-595 (1983)

43. Todd, J.T., Farley Norman, J., Mingolla, E.: Lightness constancy in the presence of specular highlights. Psychol. Sci. 15(1), 33-39 (2004)

44. Ramanarayanan, G., Bala, K., Ferwerda, J.A.: Perception of complex aggregates. ACM Trans. Graph. 27(3), 60:1-60:10 (2008)

45. Fleming, R.W., Dror, R.O., Adelson, E.H.: Real-world illumination and the perception of surface. J. Vis. 3(5), 347-368 (2003)

46. Fleming, R.W., Torralba, A., Adelson, E.H.: Specular reflections and the perception of shape. J. Vis. 4(9), 798-820 (2004)

47. O'Shea, J.P., Banks, M.S., Agrawala, M.: The assumed light direction for perceiving shape from shading. In: Proceedings of NPAR, pp. 135-142 (2008)

48. Funkhouser, T., Shilane, P.: Partial matching of 3D shapes with priority-driven search. In: Proceedings of the Fourth Eurographics Symposium on Geometry Processing, pp. 131-142 (2006)

49. Liu, R., Zhang, H.: Mesh segmentation via spectral embedding and contour analysis. In: Proceedings of NPAR, pp. 111-119 (2007)

50. Ng, R., Ramamoorthi, R., Hanrahan, P.: All frequency shadows using non-linear wavelet lighting approximation. ACM Trans. Graph. 22(3), 376-381 (2003)

51. Buatois, L., Caumon, G., Levy, B.: Concurrent number cruncher: An efficient sparse linear solver on the GPU. In: High Performance Computation Conference (HPCC). Springer, Berlin (2007) 


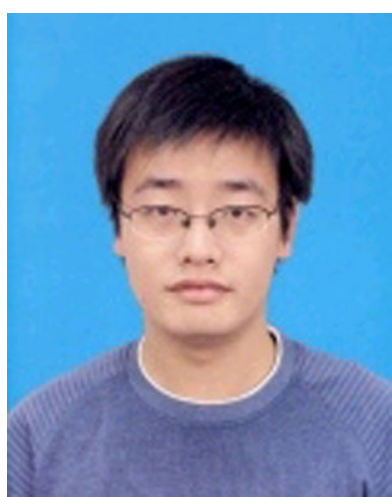

Xin Zhang is a Ph.D. candidate of the State Key Lab. of CAD\&CG at Zhejiang University, P.R. China. He received his B.E. degree in 2006 from the Department of Mathematics in Zhejiang University. His research interests include virtual reality, digital geometry processing, and perceptually-motivated rendering.

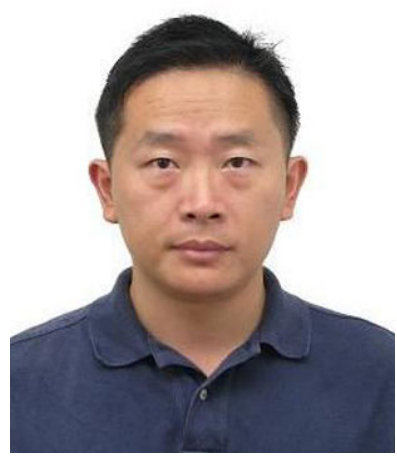

Wei Chen is a professor in State Key Lab. of CAD\&CG at Zhejiang University, P.R. China. From June 2000 to June 2002, he was a joint $\mathrm{Ph} . D$. student in Fraunhofer Institute for Graphics, Darmstadt, Germany and received his Ph.D. degree in July 2002. He was a visiting scholar at Purdue University, working in PURPL with Prof. David S. Ebert. His current research interests include scientific visualization, visual analytics, and biomedical image computing.

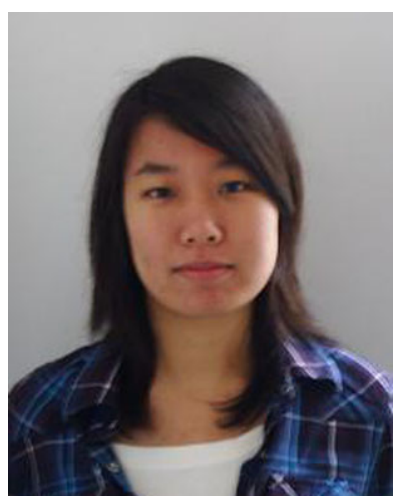

Jing Fang is now a fourth year undergraduate student at Zhejiang University, P.R. China. She majors in Computer Science and Technology, and is a member of the State Key Lab. of CAD\&CG at Zhejiang University. Her research interests include perceptually-motivated rendering and scientific visualization.

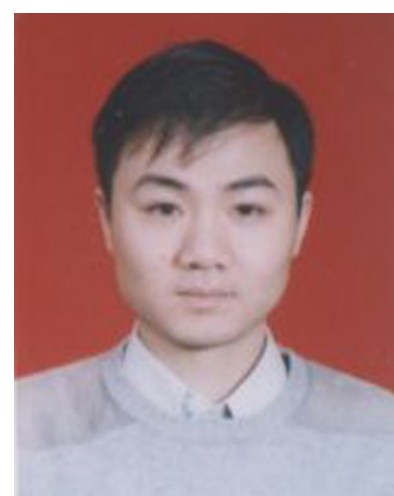

Rui Wang received the Ph.D. degree in Applied Mathematics from Zhejiang University in 2007. He is currently an assist researcher of the State Key Laboratory of CAD\&CG of Zhejiang University. His research interests include real-time realistic rendering, perceptually-motivated rendering, and virtual reality.

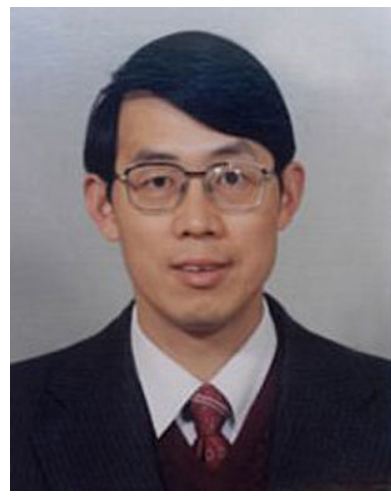

Qunsheng Peng is a professor in State Key Lab of CAD\&CG, Zhejiang University. He graduated from Beijing Mechanical College in 1970 and received his $\mathrm{Ph} . \mathrm{D}$. degree in the School of Computing Studies, University of East Anglia, UK, in 1983. His research interests include realistic image synthesis, virtual reality, biomolecule graphics, and scientific visualization. In these fields, he has authored and coauthored more than two hundred journal and conference papers. He is a member of the editorial board of several international and Chinese journals. 